TENDENCIAS

Revista de la Facultad de Ciencias

Económicas y Administrativas.

Universidad de Nariño

ISSN-E 2539-0554

Vol. XXI No. 1 - 1er Semestre 2020,

Enero - Junio - Páginas 197-220

\title{
EMPRESAS FAMILIARES: DEFINICIONES, CARACTERÍSTICAS Y CONTRIBUCIONES
}

\section{FAMILY COMPANIES: DEFINITIÓN, CHARACTERISTICS AND CONTRIBUTIONS}

\section{EMPRESAS FAMILIARES: DEFINIÇÕES, CARACTERÍSTICAS E CONTRIBUIÇÕES}

\author{
MUÑOZ MUÑOZ_Diego Fernando, MATABANCHOY TULCÁN_ Sonia Maritza, \\ GUEVARA CANCHALA_ Nayive Tatiana
}

Psicólogo egresado Programa de Psicología, Universidad de Nariño. E-mail: dife@udenar.edu.co, Colombia.

Doctora en Psicología, Universidad Católica de Argentina. Docente tiempo completo Programa Psicología, Universidad de Nariño. E-mail: somapsicologa@ yahoo.es, Colombia.

Psicóloga egresada Programa de Psicología, Universidad de Nariño. E-mail: nayivetat1234@gmail.com, Colombia.

Recibido: 1 de noviembre de 2019

Aprobado: 3 de junio de 2020

DOI: https://doi.org/10.22267/rtend.202101.133

\section{RESUMEN}

La empresa familiar se puede entender como una organización compleja, atravesada por un vínculo familiar que direcciona la política empresarial, sus objetivos y metas. En ella la propiedad, su gestión y su posterior sucesión son áreas que, clásicamente, evidencian los problemas que más comúnmente se han estudiado, y los cuales determinan la continuidad de la organización más allá de la segunda generación. Objetivo: identificar estudios realizados sobre empresas familiares desde diferentes 
disciplinas en el periodo de 2007 a 2019. Método: Se realizó bajo la metodología de revisión sistemática, mediante la búsqueda de artículos en las bases de datos Dialnet, Redalyc, Scielo e IDEA. Resultados: las áreas académicas comerciales, financieras y de mercadeo lideran la investigación relacionada a las empresas familiares. Se visualiza que la sucesión, las relaciones entre los propietarios y la relevancia de las empresas familiares en la economía en general han sido los temas más abordados, mostrándose como una oportunidad de mejora y de aporte por parte de las disciplinas interesadas en las empresas familiares. Por otro lado, la posibilidad de generar modelos de análisis de los procesos de GTH en las EF se articula como una oportunidad para responder a las necesidades específicas de este tipo de empresas.

Palabras clave: emprendimiento, nuevas empresas, recursos humanos, gestión laboral, Latinoamérica.

JEL: L26, M13, M54, O15, O54

\section{ABSTRACT}

The family business can be understood as a complex organization, crossed by a family bond that directs business policy, its objectives and goals. In it the property, its management and its subsequent succession are areas that, classically, show the problems that have been most commonly studied, and which determine the continuity of the organization beyond the second generation. Objective: to identify studies carried out on family businesses from different disciplines in the period from 2007 to 2019. Method: It was carried out under the methodology of systematic review, by searching for articles in the Dialnet, Redalyc, Scielo and IDEA databases. Results: Academic commercial, financial and marketing areas lead research related to family businesses. It is visualized that the succession, the relations between the owners and the relevance of family businesses in the economy in general have been the most discussed topics, showing themselves as an opportunity for improvement and contribution by the disciplines interested in family businesses. On the other hand, the possibility of generating models of analysis of GTH processes in the EF is articulated as an opportunity to respond to the specific needs of this type of companies.

Keywords: entrepreneurship, new companies, human resources, labor management, Latin America.

JEL: L26, M13, M54, O15, O54 


\section{RESUMO}

A empresa familiar pode ser entendida como uma organização complexa, atravessada por um vínculo familiar que direciona a política comercial, seus objetivos e metas. Nele, a propriedade, sua gestão e sua subsequente sucessão são áreas que, classicamente, mostram os problemas que foram mais comumente estudados e que determinam a continuidade da organização além da segunda geração. Objetivo: identificar estudos realizados em empresas familiares de diferentes disciplinas no período de 2007 a 2019. Método: Foi realizado sob a metodologia de revisão sistemática, buscando artigos nas bases de dados Dialnet, Redalyc, Scielo e IDEA. Resultados: As áreas acadêmica comercial, financeira e de marketing lideram pesquisas relacionadas a empresas familiares. Visualiza-se que a sucessão, as relações entre os proprietários e a relevância das empresas familiares na economia em geral têm sido os tópicos mais discutidos, mostrando-se como uma oportunidade de melhoria e contribuição pelas disciplinas interessadas nas empresas familiares. Por outro lado, a possibilidade de gerar modelos de análise de processos de GTH na EF é articulada como uma oportunidade de responder às necessidades específicas desse tipo de empresa.

Palavras-chave: empreendedorismo, novas empresas, recursos humanos, gestão do trabalho, América Latina.

JEL: L26, M13, M54, O15, O54

\section{INTRODUCCIÓN}

Según datos suministrados por el DANE, en el contexto colombiano, las pequeñas y medianas empresas representan aproximadamente $90 \%$ del potencial empresarial nacional, las cuales generan un $73 \%$ de empleo y aportan un 53\% de producción bruta de los sectores industria, comercio y servicios (Jaramillo, 2005). Es así que, como menciona Jaramillo (2005), las empresas familiares EF son fundamentales en el contexto económico nacional, ya que permiten generar empleo, ingresos, flexibilidad laboral, capacidad de adaptación y aprendizaje. Sin embargo, es curioso ver cómo, aún con la importancia mencionada, es difícil encontrar consolidados de censos actuales sobre estas unidades empresariales. 
Aunado a lo anterior, se torna relevante el estudio de las EF, siendo un tópico que, aunque referido en diversas ocasiones en la literatura científica, no parte de un consenso sobre su definición, implicaciones y características (Vélez et al, 2008). Sin embargo, posee una dinámica particular que vincula (familia, propiedad y empresa). Generando ventajas competitivas como desventajas. En lo que refiere a la gestión del talento humano Carrasco \& Sánchez (2014) sostienen que los pocos trabajos que se han identificado se centraron en él débete de determinar en qué medida se da un conjunto de mejores prácticas de recursos humanos y en qué grado son aplicadas y adaptadas a las EF.

Con base a lo anterior, el identificar aspectos de aporte hacia la conceptualización teórica y el desarrollo práctico de las EF facilitaría el desarrollo de conceptos claros de partida para futuras investigaciones. La presente investigación se enfocó en identificar estudios realizados sobre empresas familiares y los aportes realizados por distintas disciplinas sobre el tema en cuestión, tal identificación se sustenta en una necesidad de contexto referida a la no existencia de estudios de revisión sistemática sobre EF en la región; por otro lado, se sustenta en el interés de analizar las dinámicas y el funcionamientos que presentan las EF en distintos contextos, y así lograr obtener unas características comunes que permitan aportar a la consecución de un concepto claro sobre ellas.

\section{REFERENTES TEÓRICOS}

\section{Empresas Familiares}

La carencia de un concepto operativo claro sobre las EF implica que las investigaciones que respecto a ellas se realizan se sustenten con base en sus características distintivas que, por otro lado, son compartidas por distintos abordajes; por lo general estas características son: a) que la propiedad de la empresa debe estar subordinada a individuos unidos por un lazo familiar directo, de hecho o legal (matrimonio), b) que las funciones administrativas y de gerencia sean realizadas por individuos pertenecientes a la familia y c) que exista interés por la continuidad y desarrollo de la empresa (Román, 2009; Quijano, Magaña \& Pérez, 2011; Betancourt, Arcos, Torres \& Olivares, 2012) lo cual se corresponde con el modelo de los tres círculos, en donde condicionan la existencia de este tipo de empresas a la interconexión de tres sistemas: La propiedad, la familia y la empresa (Marcelino et al. 2012). 
Posiblemente la divergencia de conceptos planteados sobre la EF se explica por la interacción compleja y limítrofe de las relaciones familiares, el control que se genera a partir de ellas y la trayectoria de la EF (Quejada \& Ávila, 2016). Según Rentería y Orozco (2012) la empresa familiar se entiende como una organización permeada por un vínculo familiar, que influencia tanto las políticas de la empresa como sus intereses y objetivos. La familia permite integrar una asociación interfamiliar para conformar distintos tipos de sociedad empresarial (Páez, 2014). Este tipo de organización posee la ventaja del compromiso de los integrantes de la familia con la empresa, basado en la confianza. Por otro lado, los conflictos entre familiares afectan las dinámicas de la empresa, generando tensión y poniendo en riesgo la continuidad de la EF. Entre las tenciones comúnmente mencionadas se destaca la resistencia al ingreso de socios no familiares, la preocupación por la sucesión y la persistencia de la cultura corporativa con el deseo de mantener la empresa (Sandoval \& Guerrero, 2010; Marcelino, Baldazo, \& Valdés, 2012).

En tanto a características particulares de las empresas familiares se mencionan: Las decisiones empresariales, las cuales están a cargo de la familia, aunque las responsabilidades y la dirección son asumidas solo por algunos miembros de esta; las relaciones familiares, las cuales interactúan con la actividad empresarial y ejercen influjo bidireccional. Por otra parte, el mercado de la empresa, su marco legal y su cultura organizacional son aspectos que generan variaciones en la EF (Rentería \& Orozco, 2012). Aunado a lo anterior la dificultad de poder establecer y conformar su propio talento humano en las EF influye tanto en su desempeño organizacional como en los valores con los que se identifican sus miembros (Sandoval \& Guerrero, 2010). Otros aspectos que se mencionan son la concentración del poder, la falta de delegación de funciones, de tecnología, de planeación a corto, mediano y largo plazo y los fallos en la comunicación interna (Jaramillo, 2005).

Con relación a la gestión del talento humano Jaramillo (2005) menciona que, debido a la ventaja competitiva que representa para la organización el contar con un adecuado talento humano, se ha generado un aumento en la inversión de los procesos de selección, formación, compensación y evaluación. Tanto en las pequeñas como en las medianas empresas existe la presencia de una actitud de rechazo hacia nuevas metodologías y sistemas de gestión de recursos humanos; pero, por otra parte, las empresas que lideran el talento humano de una manera efectiva aseguran el cumplimiento de la 
misión de la empresa (Jaramillo, 2005). De la misma manera, Carrasco \& Sánchez (2014) mencionan que la gestión del recurso humano repercute en el equilibrio de las practicas que determinan la satisfacción, motivación e implicación de los empleados y familia en el negocio. Hernando (2014) plantea que los obstáculos con los que puede encontrarse la gestión humana en la EF son: a) la insuficiente dedicación de los directivos al estudio del talento en la empresa, b) la ausencia de compromiso de los integrantes de la empresa, c) la inconveniencia de identificar a los mejores desempeños y d) la incongruencia entre la gestión del talento y los objetivos empresariales. En este sentido Carrasco \& Sánchez (2014) mencionan que los trabajos existentes sobre EF no han profundizado en las prácticas de recursos humanos que permitan identificar, aprovechar, desarrollar y proteger mejor el capital humano de las EF.

\section{III.METODOLOGÍA}

\section{Tipo de estudio}

La presente investigación es una Revisión sistemática. Según Beltrán (2005) la revisión sistemática es un estudio integrativo, observacional, retrospectivo, secundario, en el cual se combinan estudios que examinan la misma pregunta. Para el presente estudio, se utiliza tal metodología en pro de abarcar los estudios que se hayan realizado respecto al tópico de empresas familiares, realizando la búsqueda en 4 bases de datos, a saber: IDEA, Dialnet, Scielo y Redalyc.

\section{Criterios de inclusión}

Los criterios de inclusión tenidos en cuenta en la presente revisión sistemática fueron los siguientes: a) Artículos encontrados mediante las palabras "Empresas familiares" o "family bussines", b) Artículos completos, c) Artículos en idioma español e inglés, d) Los artículos deben estar publicados en un lapso comprendido entre 2007 y 2019 y e) Artículos nacionales e internacionales.

\section{Criterios de Exclusión}

Los criterios de exclusión tenidos en cuenta en la presente revisión sistemática fueron los siguientes: a) Artículos de tipo ensayo o reflexión teórica, b) El tema principal no sea empresas familiares y c) Artículos que no estén completos, o que su acceso sea limitado. 


\section{Procesos de búsqueda}

Para la revisión bibliográfica, se tuvo en cuenta 4 bases de datos de libre acceso, con el fin de tener una mayor cobertura de la temática desde una perspectiva interdisciplinar. Las bases de datos empleadas fueron: Dialnet, Redalyc, Scielo, IDEA.

\section{Procedimiento}

Se realizó una selección de los artículos encontrados, escogiendo aquellos que cumplían con los criterios de inclusión anteriormente señalados. Posteriormente la información de tales artículos fue vaciada en una matriz de información para facilitar el recate de la información más relevante de los mismos. Es importante mencionar que la matriz usada para la presente revisión fue diseñada teniendo en cuenta las recomendaciones y el protocolo de la metodología PRISMA-P para revisiones sistemáticas (Moher, Shamseer, Clarke, Ghersi et al., 2015).

\section{Codificación de los resultados}

De cada uno de los trabajos se extrae la siguiente información: I) Autor/es y año de publicación, II) Metodología del trabajo: al presentarse artículos desde distintas disciplinas, la forma metodológica de acercamiento investigativo cobro relevancia como aspecto a revisar, III) Muestra, IV) Técnicas e instrumentos, VI) Sector, VII) Definición del concepto. Referido al concepto de empresa familiar del cual partieron los autores para la realización de la investigación, VIII) Principales resultados, IX) Limitaciones, X) Recomendaciones.

\section{IV.RESULTADOS Y DISCUSIÓN}

Se encontraron 73 artículos mediante los criterios de inclusión, de los cuales 8 fueron eliminados por criterios de exclusión, ya que de dos solo se tenía acceso a su resumen, mientras que los restantes eran reflexiones teóricas. En el presente apartado se recogen aspectos tales como el país de procedencia de los artículos, su año de publicación, la disciplina desde la cual se abordó y el sector donde se realizó la investigación (Ver Tabla 1). Se rescata en este punto el hecho de que Colombia lidere el campo de investigación de las EF en cuanto a Iberoamérica se refiere. 


\section{Tabla 1}

Artículos por país

\begin{tabular}{lrr}
\hline \multicolumn{1}{c}{ País } & Número de artículos & \multicolumn{1}{l}{ Total } \\
\hline Argentina & 1 & 1,5 \\
Brasil & 4 & 6,2 \\
Colombia & 27 & 41,5 \\
Cuba & 1 & 1,5 \\
Ecuador & 5 & 7,7 \\
España & 6 & 9,2 \\
México & 17 & 26,2 \\
Uruguay & 1 & 1,5 \\
Venezuela & 3 & 4,6 \\
Total & 65 & 100,0 \\
\hline
\end{tabular}

Fuente: elaboración propia.

En cuanto a las publicaciones que se han realizado desde el año 2007 hasta el 2019 (años tomados como criterio de búsqueda) se pudo encontrar que el número de investigaciones tuvo un incremento en el año 2011 respecto a años tanto posteriores como anteriores, contando con 11 artículos (16,9\%), seguido del año 2016 con 9 artículos (13,8\%) y el año 2012 con 6 artículos (9,2\%). El año 2019 presenta poca productividad académica en tanto EF, contando con 2 artículos $(3,1 \%)$.

La siguiente variable retomada fue el sector al que pertenecían las EF documentadas en los artículos. Se encontró que en más de la mitad de los casos no se especificaba el sector, encontrándose 43 artículos $(66,2 \%)$ en los cuales no se encuentra información al respecto. Por otro lado, 10 artículos $(15,4 \%)$ mencionan que las investigaciones se realizaron en EF multisectorial, refiriéndose a que abarcaron en sus muestras empresas tanto de productos como de servicios. Por otro lado, existen 12 artículos que contienen sectores de investigación muy específicos y diferentes entre sí, como la agroindustria, la Biotecnología y la Hostelería, por dar algunos ejemplos (Ver Tabla 2). 


\section{Tabla 2}

Sectores de las EF estudiadas

\begin{tabular}{lcc}
\hline & Frecuencia & Porcentaje \\
\hline Agroindustria & 1 & 1,5 \\
Alimentos & 1 & 1,5 \\
Biotecnología & 1 & 1,5 \\
Comercio y servicios & 1 & 1,5 \\
Constructoras de vías terrestres & 1 & 1,5 \\
Exportaciones & 1 & 1,5 \\
Iluminación & 1 & 1,5 \\
Manufactura & 1 & 1,5 \\
Multisector & 10 & 15,4 \\
No especifica & 43 & 66,2 \\
Sector de Hostelería & 1 & 1,5 \\
Textil & 1 & 1,5 \\
Transporte & 1 & 1,5 \\
Turismo & 1 & 1,5 \\
Total & 65 & 100,0 \\
\hline
\end{tabular}

Fuente: elaboración propia.

Con respecto a las disciplinas desde las cuales se abarcan los artículos, se encontró que la disciplina que mayores aportes en tanto productividad bibliográfica ha proporcionado es la Administración de Empresas, contando con 37 artículos (56,9\%), seguida por Economía con 12 (18,5\%). Se encuentra, además, que las disciplinas de Comercio y contaduría realizan un aporte importante dentro del material bibliográfico recabado, teniendo $3(4,6 \%)$ y $4(6,2 \%)$ artículos respectivamente (Ver Tabla 3 ). 


\section{Tabla 3}

Artículos por disciplina

\begin{tabular}{lrr}
\hline & Frecuencia & Porcentaje \\
\hline Administración Empresas & 37 & 56,9 \\
Comercio & 3 & 4,6 \\
Contaduría & 4 & 6,2 \\
Economía & 12 & 18,5 \\
Historia & 1 & 1,5 \\
Mercadeo & 1 & 1,5 \\
No especifica & 4 & 6,2 \\
Psicología & 2 & 3,1 \\
Trabajo social & 1 & 1,5 \\
Total & 65 & 100,0
\end{tabular}

Fuente: elaboración propia.

De esta manera, el objetivo del estudio fue identificar los estudios realizados sobre empresas familiares desde diferentes disciplinas, teniendo en cuenta sus aportes a conceptualización, características y aportes. Preliminarmente, la cantidad de artículos encontrados parece indicar que las EF son objetos de investigación considerados importantes, y se adelantan esfuerzos para caracterizarlas y comprender su funcionamiento. Es probable que la cantidad de estudios por país pueda deberse tanto a la proporción de la economía representada por tales unidades empresariales como a las líneas de investigación académicas existentes en los países (Colombia cuenta con una línea fuerte liderada por la Universidad del Valle). Por otro lado, es importante tener en cuenta la diversidad de metodologías en el estudio de las EF pueden ser entendidas desde el marco de la interdisciplinariedad, que en el caso cuenta con la meta de aportar en el desarrollo y fortalecimiento de las EF sobre la base de múltiples aportes a distintos niveles teórico-prácticos (Rentería y Orozco, 2012). 


\section{Definiciones Encontradas}

En la Tabla 4 se muestran las definiciones de empresa Familiar encontradas en la revisión. Se debe mencionar que se seleccionó los conceptos de EF que contaban con una definición clara y operativa del mismo.

\section{Tabla 4}

\section{Definiciones de empresas familiares}

Autor (es) Concepto desarrollado

Una empresa familiar es aquella en la cual miembros de una misma familia

Fuentes, Vallejo poseen una parte de la propiedad que les permite influir notablemente en la \& Martínez dirección y control estratégico del negocio y en la que además se comparte un (2007) deseo de continuidad en manos de la siguiente generación.

Román (2009) La empresa familiar es aquella que logra conjugar los siguientes elementos: a) control sobre la propiedad (15\% o más) por parte de dos o más miembros de una familia o asociación de familias; b) influencia estratégica de los integrantes de la familia en la dirección de la empresa; c) interés por las buenas relaciones familiares; d) interés en la continuidad de la empresa de generación a generación.

Bolaños, Pantoja La EF es aquella en la que la propiedad (o un fragmento) pertenece a un grupo

\& Jiménez (2009) familiar, en la cual haya (cuando menos) dos miembros de la familia implicados en el desarrollo de actividades dentro de la organización.

Zeron, Mendoza Se considera como una EF la organización en la cual hay injerencia directa en

\& Lara (2009) sus actividades y propiedad por parte de una agrupación familiar.

Quijano, Magaña La EF se caracteriza por la propiedad y participación de una familia en las \& Pérez (2011) actividades que desde ella se realizan.

Macías \& Las empresas familiares son propiedad de una familia y son controladas por Ramírez (2011) ésta. En este caso, la unidad básica de cohesión social llamada familia, sirve también como unidad básica para la organización económica. 
Betancourt, La EF es una unidad empresarial manejada por integrantes de grupo familiar,

Arcos, Torres \& que tienen como objetivo la continuidad del negocio bajo el control del mismo Olivares (2012) núcleo.

Marcelino, La Empresa Familiar se define como aquella en la que varios miembros de una Baldazo y Valdez misma familia participan como propietarios o gerentes, en forma simultánea o (2012) durante algún tiempo.

Arenas \& Rico Las empresas familiares se articulan bajo tres aspectos, correspondientes con: (2014) el control empresarial, el poder familiar ejercido y la intención de sucesión.

Angulo (2016) Se entiende por empresa familiar a aquella organización en donde la propiedad, la dirección y el control de la misma se concentran en un núcleo familiar.

Fuente: elaboración propia.

Con respecto a las definiciones es pertinente mencionar los aspectos recurrentes encontrados en ellas. Inicialmente todas las definiciones confluyen en el hecho de que existe un control o administración de la empresa o negocio por parte de una familia o de un grupo de familiares; estos últimos poseen un poder de decisión en todos los ámbitos de la empresa (ya sea en temas de contratación, de gerencia, y en general las funciones empresariales básicas). Por otro lado, un aspecto interesante encontrado en las definiciones se refiere a la intencionalidad o deseo, por parte de la familia, de continuar con la EF en una siguiente generación.

En consonancia, a lo largo de los artículos revisados, se encuentra que no existe una definición uniforme que abarque las características de la empresa familiar (Vélez et al, 2008). Aun así, la literatura existente respecto de las EF converge dos aspectos: la existencia de un lazo consanguíneo o de hecho que una a los integrantes de la empresa, y el hecho de que cada participante pueda tomar decisiones y ejercer una influencia sobre la organización (Román, 2009; Marcelino et al. 2012).

\section{Resultados por subcategorías de análisis}

Para empezar, se realizó el análisis de las principales recomendaciones y limitaciones expuestas en los trabajos revisados, las cuales se muestran a continuación en la Tabla 5: 


\section{Tabla 5}

Resultados por subcategorías

Resultados por subcategorías

\begin{tabular}{|c|c|}
\hline Sub-Categoría & Definición del resultado \\
\hline $\begin{array}{l}\text { Tipos de estudio } \\
\text { de las } \\
\text { investigaciones }\end{array}$ & $\begin{array}{l}\text { En cuanto a los tipos de estudio que se usaron en los artículos, se } \\
\text { encuentran: } \\
\text { Estudios interpretativos: } 10,4 \% \text {. } \\
\text { Estudios explicativos: } 8,3 \% \text {. } \\
\text { Estudios exploratorios y descriptivos: } 43,8 \% \text {. } \\
\text { Análisis Clúster: } 2,1 \% \text {. } \\
\text { Asesorías empresariales: } 2,1 \% \text {. } \\
\text { Investigación acción: } 2,1 \% \text {. } \\
\text { Análisis de caso: } 12,5 \% \text {. } \\
\text { Reflexiones teóricas: } 18,7 \% \text {. }\end{array}$ \\
\hline Recomendaciones & $\begin{array}{l}\text { A partir de la revisión de los distintos artículos encontrados en el } \\
\text { presente ejercicio, se identifica como recomendaciones comunes entre } \\
\text { artículos: la necesidad de propender por una relación de equilibrio entre } \\
\text { dinámica familiar y organizacional, para tal aspecto, algunos de los } \\
\text { artículos encontrados mencionan los protocolos familiares como un } \\
\text { medio para poder equilibrar esta relación. Se menciona además que } \\
\text { existen diferencias geográficas y culturales que determinan en gran } \\
\text { manera las características de las EF, por lo cual se considera pertinente } \\
\text { evaluar esas condiciones sociodemográficas y su incidencia en el } \\
\text { desarrollo y mantenimiento de las empresas familiares. }\end{array}$ \\
\hline Limitaciones & $\begin{array}{l}\text { Se logró encontrar, entre las limitaciones más mencionadas en los } \\
\text { artículos, que no existe una única definición teórico-empírica de las } \\
\text { empresas familiares. Por otro lado, se refiere como limitación el alcance } \\
\text { del estudio, ya que, en gran medida, estos son de tipo exploratorio, } \\
\text { reflexivo o descriptivo. Se aduce además que existe una limitación en }\end{array}$ \\
\hline
\end{tabular}


tanto instrumentos psicométricos desarrollados para esta población, en los cuales se tenga en cuenta sus características particulares.

\section{Sub-Categorías emergentes}

Características del La edad está enlazada con el tiempo de la empresa familiar, lo cual sucesor y genera que un cierto grado de incertidumbre y a la vez de preocupación predecesor: edad por lo que puede suceder con las siguientes generaciones. Por otro lado, la edad del predecesor presenta relevancia en tanto las atribuciones causales que esta adquiere (mayor edad como sinónimo de sabiduría y madurez) y que hacen que los procesos de sucesión se dificulten.

Sucesor de las Su importancia radica en su papel fundamental para el seguimiento y empresas progreso empresarial. Por lo tanto, la adecuada elección del sucesor adquiere relevancia; en la misma línea, una de las principales crisis que se encuentran en las EF es la inexistencia de protocolos claros de sucesión en este tipo de empresas que empiezan a conformarse.

Conflictos La organización y capacidad gerencial son las problemáticas que más generan enfrentamientos en las empresas que se puede destacar.

Plan de sucesión Este punto se articula como uno de los de mayor consenso como aspecto relevante entre los artículos. En él la propiedad y la dirección de la empresa familiar se ponen en juego al momento de pensar en dicha el proceso de transición entre administradores de la empresa. Se debe mencionar a su vez que es un proceso de suma relevancia, pero de igual manera esta situación es crítica ya que por medio de esta se dará la continuación a los procesos que se han adelantado en la organización, por lo cual este proceso es determinante para que la EF no sucumba en la 2 o 3 generación.

Vinculación La cual puede darse a una edad temprana lo cual favorece a un apego más fraterno y una mejor sucesión; por otra parte, puede darse a una edad más tardía por lo tanto se da un manejo y un proceso de manera diferente. 
El poder que La influencia familiar en la organización tiene implicaciones en los ejerce la familia lineamientos y dirección que tendrá la empresa para poder seguir el sobre la empresa funcionamiento que la caracteriza.

Financiamiento Las EF suelen tener una aceptable estabilidad económica debido a que estas cuentan y se gestionan en sus primeros años con recursos provenientes del arreglo familiar existente. Dicha estabilidad se mantiene a partir de su autofinanciamiento y a la resistencia a incluir a personas ajenas a su familia en los primeros momentos de la EF.

Gestión de la En la gestión organizacional de las EF el concejo familiar adquiere Organización relevancia, ya que se encarga de la regulación de la empresa; por otra parte, según los autores una buena gestión organizacional de las EF implicaría la existencia de un protocolo familiar, el cual hace referencia a las normas establecidas dentro de la empresa. Se evidencia la inexistencia de estos protocolos en la mayoría de EF estudiadas.

Amenazas que Entre las amenazas mencionadas se encuentra la globalización, ya que, pueden afectar a según lo encontrado, puede generar una serie de factores las empresas desestabilizantes tanto político, tecnológicos y financieros, los cuales familiares pueden afectar de manera directa el mercado de las diferentes empresas (entre ellas las familiares). Por otro lado, este aspecto también se reconoce como una oportunidad de expansión y de apertura a nuevos mercados por parte de las EF.

Factores que Se trae a colación factores como el cuidado del patrimonio, el desarrollo influyen en la profesional y conservación de la unidad familiar y la influencia que la participación de la mujer puede ejercer en ellos. Por otro lado se menciona que la mujer en las participación de la mujer en la EF provee una buena comunicación entre empresas quienes conforman la unidad familiar, lo cual lleva a una buena familiares comunicación y una dinámica fluida en los aspectos tanto administrativos como relacionales en las EF.

Factores para el La unidad y el compromiso de la familia con sus empresas constituyen éxito la ventaja competitiva de las que son exitosas, al igual que la confianza 
reinante en la familia. A partir de dimensiones estructurales, cognitivas y relacionales pueden conducir a ventajas competitivas basadas en el desarrollo de procesos organizativos y capacidades respecto al acceso y uso de la información.

Oportunidades de Una oportunidad para el desarrollo es la internacionalización. Las mejora empresas que se internacionalizan son percibidas abiertas y experimentadas, con buena reputación en el exterior, lo que a su vez provoca que sigan avanzando en su proyección internacional. Por el contrario, las empresas domésticas, con ninguna presencia, ni reputación en mercados exteriores, no llegan a los niveles de reconocimiento que consiguen las primeras.

Aun así, es importante mencionar que la internacionalización es un objetivo a largo plazo. En las etapas iniciales de las EF, los esfuerzos se centran en no dejar decaer la empresa; cuando una EF ya se ha establecido en términos económicos, administrativos y de mercado, la internacionalización o apertura de nuevos mercados puede ser el paso para seguir.

Fuente: elaboración propia.

Respecto a las características de las EF, como aspectos que le dan particularidad a sus dinámicas se encuentra la sucesión: la sucesión es el proceso en el cual quien está a la cabeza de la organización debe ceder la dirección de la misma. En las EF, el proceso no se da desde protocolos establecidos, sino que el proceso se da con los tiempos establecidos por el mismo director. Esto implica que, a veces, quien está al mando no tome la decisión de dejar su patrimonio a un sucesor, ya que considera que todo lo que ha ganado podría "perderse", razón por la cual algunas EF quedan acéfalas, y el proceso se da de manera abrupta, lo que probabiliza el decaimiento de la organización (Romano \& Redondo, 2013; Gómez, López \& Betancourt, 2008; Quijano, Magaña, \& Perez, 2011).

Otro punto que se resalta en el análisis es el hecho de que, en cuanto a gestión de la organización se refiere, la familia se encarga de la regulación de la empresa, en temas como la adhesión de nuevos empleados, normatividad y reglamentación de las relaciones laborales; el meollo se encuentra en el 
hecho de que el establecimiento de esas pautas de comportamiento intralaboral no se da a manera de un protocolo, sino más bien se presenta como acuerdos verbales que, en determinadas situaciones, pueden afectar la dinámica de crecimiento empresarial (Arenas \& Rico, 2014; Correa, Restrepo, \& Acevedo, 2007). En ese orden de ideas, y como mencionan Arenas y Rico (2014) la existencia de protocolos claros y bien establecidos es un factor determinante en el afrontamiento de crisis en la empresa familiar, tanto para las relaciones dentro del trabajo, como para los procesos de sucesión (Gómez, Zapata \& Betancourt, 2016; Cervera, Montoya \& Montoya, 2012; Betancourt, Gómez \& López, 2011).

Los conflictos intrafamiliares afectan las dinámicas empresariales, provocando tensión generando riesgo en la continuidad de la EF. Así, un aspecto particular dentro de la revisión es la mención que se hace a la influencia de la mujer en el cuidado del patrimonio, el desarrollo profesional y la conservación de la unidad familiar: la participación de la mujer en la EF provee una buena comunicación entre quienes conforman la unidad familiar, dinamizando las relaciones internas en las EF (Gómez, López \& Betancourt, 2011).

Los valores (algunos mencionados como el respeto, la honradez y el orgullo por la empresa) que son compartidos por los administradores familiares y los no familiares generan tanto motivación, cooperación y compromiso, lo cual se refleja en culturas fuertes y bien administradas que incrementan la eficiencia organizacional de las empresas familiares, impactando en la continuidad y en el éxito de las mismas (Romero, 2009; Cervera, Montoya \& Montoya, 2012; Esparza \& García, 2011).

\section{Aportes a la Gestión del Talento Humano en las EF}

Se retoman aportes encontrados en tres de los artículos revisados, en los cuales se retoma la gestión del talento humano en las EF. Es necesario mencionar que estos aportes se dan desde la disciplina de Administración de empresas (Ver Tabla 6): 


\section{Tabla 6}

Reflexiones sobre los procesos de gestión del talento humano en la empresa familiar

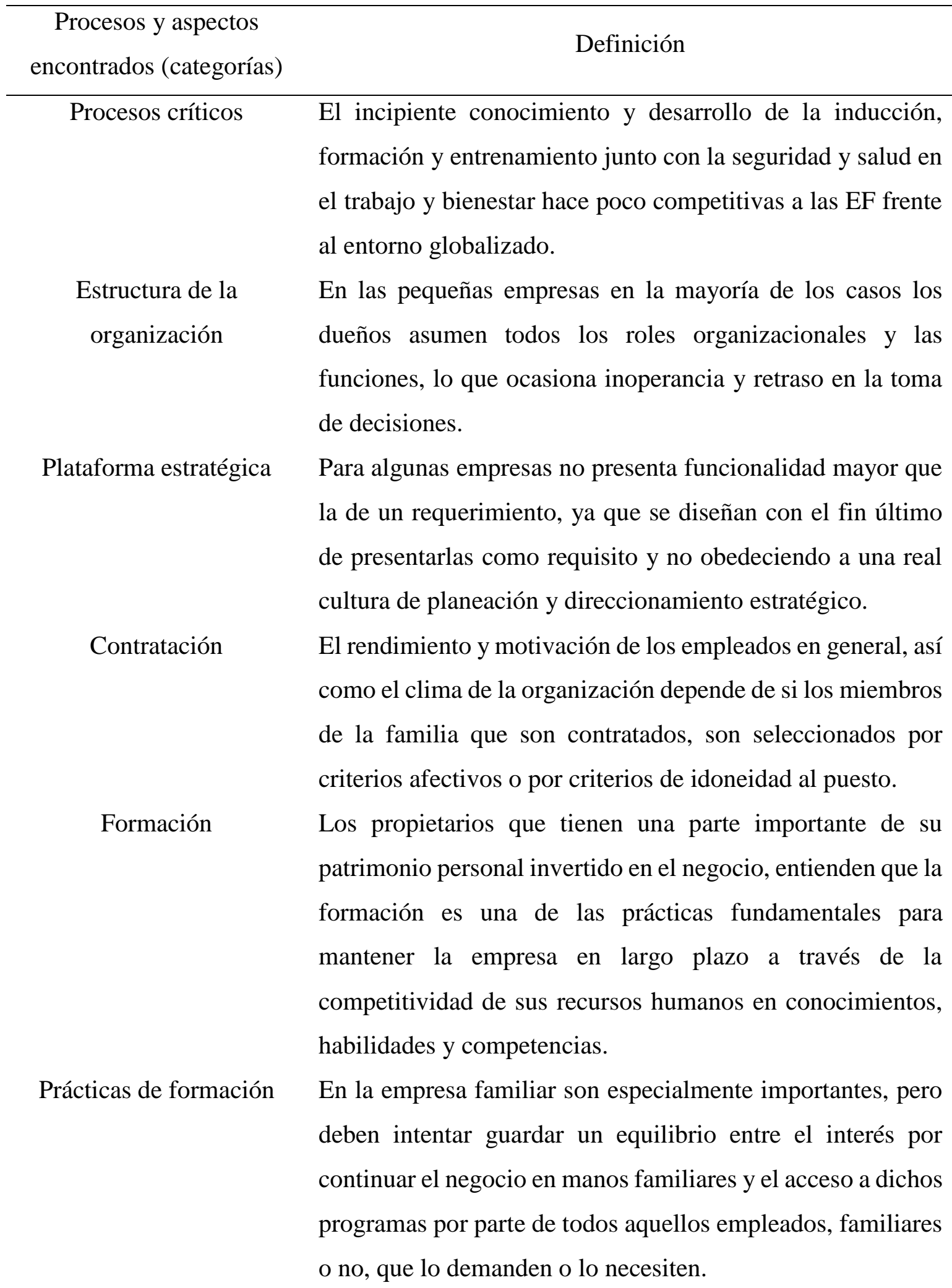


Retribución y recompensa Dentro de la empresa familiar se ha encontrado diferencias entre unos tipos y otros de empleados. Por ejemplo, los incentivos suelen tener un menor peso en el salario total para los empleados familiares frente a los no familiares y, a la inversa, cuando se trata del salario fijo. Además, en caso de no ser propietarios de la empresa, las recompensas extra dadas a familiares, se fijan en muchas ocasiones más por criterios emocionales y altruistas que por criterios de eficiencia en el trabajo.

Políticas de recursos humanos

Desafío
Del equilibrio entre familia y empresa depende que las políticas de recursos humanos se orienten en mayor o menor medida a la aplicación de principios de equidad y profesionalidad para mantener un capital humano motivado $\mathrm{y}$ eficiente que aporte valor a la empresa.

Las empresas familiares enfrentan a las organizaciones bien establecidas en lo referido a gestión del talento humano. Se debe lograr establecer relaciones fuertes y vínculos emocionales entre la EF y los empleados, dadas las nuevas características de los vínculos laborales.

Por otro lado, la comprensión sobre los procesos de GTH en las EF debe partir de un modelo inductivo, que se genere a partir de las particularidades que poseen este tipo de empresas, y que permita comprender las dinámicas específicas que en la interacción organizacional se dan.

Fuente: elaboración propia.

Con respecto a los procesos de talento humano, se menciona que el hecho de que estos se hayan empezado a implementar de manera incipiente hace que las EF sean poco competitivas frente al entorno globalizado. De la misma manera, el capital del que dispone la EF influye en la presencia de áreas de recursos humanos dentro de su estructura, lo cual explica el hecho de que en muchos casos 
los dueños asumen los roles organizacionales y las funciones del área de recursos humanos. Además, se menciona que el equilibrio entre familia y empresa incide en que las políticas de recursos humanos se orienten en mayor o menor medida a la aplicación de principios de equidad y profesionalidad para mantener un capital humano motivado y eficiente que aporte valor a la empresa. Finalmente, se debe mencionar que, dadas las nuevas características de los vínculos laborales, se debe lograr establecer relaciones fuertes y vínculos emocionales entre la EF y los empleados (Carrasco \& Sánchez, 2014; Romano \& Redondo, 2016).

\section{CONCLUSIONES}

Si bien las definiciones sobre las EF difieren en su estructura específica, la generalidad de las características de lazo familiar, vínculo organizativo y deseo de continuidad son comunes en los abordajes teóricos y empíricos adelantados.

En lo referido a las características de las EF, un aspecto que adquiere relevancia en su estudio es el proceso de sucesión, en tanto es un aspecto que determinan la continuidad de las EF, y a la investigación, protocolos y estrategias que surjan a partir de este aspecto permitirá un equilibrio armónico entre la unidad empresarial y los miembros familiares.

Con respecto a la Gestión del Talento humano, lo encontrado permite establecer la importancia de la implementación de áreas de recursos humanos en las EF, como manera de impulsar el desarrollo organizacional y potenciar las variables influyentes en el crecimiento empresarial. Se mencionan factores que afectan su puesta en marcha, como lo son la falta de capital, el hecho de que en algunas EF el área de talento humano cumple cualquier función excepto la de gestionar el potencial humano de la misma, y la situación de que, por lo general, las decisiones respecto a los procesos de la gestión del TH las realiza quien está a la cabeza de la EF. Del mismo modo, se hace necesaria una comprensión de los procesos de GTH que surja a partir del análisis de las dinámicas particulares de las empresas familiares, de manera inductiva, lo cual potencie el alcance de los de la organización se puedan realizar, y que tales aportes permitan responder a las necesidades y oportunidades de cada EF.

Es relevante mencionar que la investigación acerca de la participación de la mujer en el crecimiento de su empresa familiar, en la unidad y armonía familiar y en el compromiso que ella misma siente por 
su empresa ha adquirido importancia a través de los años, esto conjunto a la tendencia creciente de los estudios de género que se publican en revistas. De tal manera, el análisis de la participación e interacción de la mujer en el sistema de la EF adquiere relevancia en tanto provee de información relevante para la comprensión de la relación y mediación que llega a generar en la empresa, como en el hecho de propiciar un campo fructífero en la investigación de género de la dinámica organizacional familiar.

La poca bibliografía existente desde la psicología organizacional y del trabajo con respecto a las EF representa una oportunidad de investigación y un área de vacío que debe ser explorada en estudios posteriores, ya que el aporte que puede brindar la POT a las EF constituye un punto de inflexión en las aproximaciones referidas a variables organizacionales, procesos de sucesión y conflictos familiares, en procura del desarrollo y mantenimiento de las EF. Aun así, se logra observar cómo tópicos tales como las relaciones familiares (desde una perspectiva holista) y la importancia de la gestión del Talento Humano son temas que empiezan a tener relevancia en el marco investigativo de las EF, y se articulan como potenciales y campos de aporte desde la Psicología, y en especial de la POT, con lo cual se pueda proponer modelos de intervención en pro de la mejora de los aspectos blandos, y fortalecimiento de las características que estén solidas dentro de la misma: todo lo anterior en búsqueda de que las empresas no decaigan en sus primeras generaciones y lleguen a consolidarse como EF generadoras de empleo y dinamizadoras de la economía regional, respondiendo al llamado de responsabilidad social y aporte regional que se promulga desde todas las disciplinas.

Con lo anterior se evidencia que el campo de investigación respecto de las EF ha sido fructífero, aunque no es un espacio terminado o completo. Las distintas disciplinas que tengan injerencia en el campo de las organizaciones pueden proveer un conocimiento más nutrido respecto de las particularidades y dinámicas de las EF, y a partir de la base de tal conocimiento proporcionar estrategias que permitan la mejora continua de las EF, en aras de potenciar el crecimiento de un tipo de organización que puede proveer estabilidad económica y social a una región. 


\section{REFERENCIAS}

(1) Angulo, K. (2016). La empresa familiar una temática en desarrollo. Oikos Polis Revista latinoamericana de Ciencias Económicas y Sociales, 1(1), 175- 198.

(2) Arenas, H. \& Rico, D. (2014). La empresa familiar, el protocolo y la sucesión familiar. Estudio Generales, 30(132), 252-258.

(3) Beltrán, O. (2005). Revisiones sistemáticas de la literatura. Revista Colombiana de Gastroenterología, 20(1),60-69.

(4) Betancourt, A., Arcos, S., Torres, A. \& Olivares, L. (2012). Empresas Familiares. Revista Académica de Investigación, (9). Recuperado de: http://www.eumed.net/rev/tlatemoani/09/emvb.pdf.

(5) Betancourt, J., Gómez, G. \& López, M. (2011). Aproximación a los factores que influyen en la visión del patrimonio en las empresas familiares colombianas. Estudios Gerenciales, 27(120), 165-183.

(6) Bolaños, S. Pantoja, M. \& Jiménez A. (2009). Emprendimientos locales: El caso de las empresas familiares en el Sur Occidente Colombiano. TEC Empresarial, 3(3), 27-37. Recuperado de: https://revistas.tec.ac.cr/index.php/tec_empresarial/article/view/654

(7) Carrasco, A. \& Sánchez, G. (2014). "El capital humano en la empresa familiar: un análisis exploratorio en empresas españolas”. Revista FIR, FAEDPYME International Review. 3(5), 19-29.

(8) Cervera, X., Montoya, I. \& Montoya, L. (2012). Factores de éxito en el relevo generacional en empresas de familia: estudio de caso. Punto de vista, 4(6), 39-65.

(9) Correa, A., Restrepo, L. \& Acevedo, V. (2007). Más allá del protocolo de familia: un abordaje sistémico con la familia empresaria. Pensamiento Psicológico, 3(9), 79-100.

(10) Esparza, J. \& García, D. (2011). La cultura de las empresas familiares turísticas mexicanas y su influencia en la gestión estratégica. Cuadernos de Administración, 24(42), 295-313.

(11) Fuentes, G., Vallejo, M. \& Martínez, R. (2007). Factores a tener en cuenta en la expansión internacional de la empresa familiar. Investigaciones Europeas de Dirección y Economía de la Empresa, 13(2), 75-96.

(12) Gómez, G., López, M. \& Betancourt, J. (2008). Estudio exploratorio de los factores que influyen en la selección de un mecanismo de sucesión patrimonial en las empresas familiares colombianas. Cuadernos de Administración, 21(37), 269-292.

(13) Gómez, G., Zapata, N \& Betancourt, J. (2016). Gobierno Corporativo. Prácticas sugeridas e implementadas por empresas familiares y no familiares colombianos. Entramado, 12(2), 12-29.

(14) Hernando, M. (2014). Un modelo de control de gestión para la pequeña empresa familiar en España: especial referencia a los activos intangibles. Universidad de Alcalá. Madrid. España. 
(15) Jaramillo, O. (2005). Gestión del talento humano en la micro, pequeña y mediana empresa vinculada al programa Expopyme de la Universidad del Norte en los sectores de confecciones y alimentos. Pensamiento \& Gestión, (18) ,103-137.

(16) López, M., Gómez, G., \& Betancourt, J. (2011). Factores que influyen en la participación de la mujer en cargos directivos y órganos de gobierno de la empresa familiar colombiana. Cuadernos de Administración, 24(42), 253-274.

(17) Macías, V. \& Ramírez, D. (2011). Sucesión en empresas familiares. Análisis desde la teoría de la agencia. Caso: Caldas. Criterio Libre, 9(15), 193-212.

(18) Marcelino, M. \& Baldazo, F. \& Valdés, O. (2012). El método del estudio de caso para estudiar las empresas familiares. Pensamiento \& Gestión, (33), 125-139.

(19) Moher, D., Shamseer, L., Clarke, M., Ghersi, D., Liberatî, A., Petticrew, M., Shekelle, P., Stewart, L \& Group, prisma-p (2015). Preferred reporting items for systematic review and meta-analysis protocols (prisma-p) 2015 statement. Systematic Reviews, 4, (1), 1-9.

(20) Páez, Y. (2014). Las empresas familiares y la supervivencia de estas, dentro de las primeras tres generaciones dados los conflictos que se presentan en la sucesión de poder y manejo de capital. Universidad Militar Nueva Granada. Santafé de Bogotá, D. C.

(21) Quejada, R. \& Ávila, J. (2016). Empresas familiares: Conceptos, teorías y estructuras. Rev. esc.adm.neg, (81), 149-158.

(22) Quijano, R., Magaña, D. \& Pérez, C. (2011). Pertenencia y compromiso: factores relevantes en la transición generacional de empresas familiares. Caso constructoras Campeche, México. Estudios Gerenciales, 27(121), 99-113.

(23) Rentería, E. \& Orozco, A. (2012). Empresa de familia: Relaciones y conflictos en la transición generacional. Cali, Colombia: Editorial Bonaventurana.

(24) Román, R. (2009). Una perspectiva heterodoxa sugerida para el estudio de las empresas familiares en Colombia. Estudios gerenciales, 25(112), 101-129.

(25) Romano, C. \& Redondo, J. (2013). Planificación de la sucesión en una empresa familiar de pequeño porte. Aplicación de la investigación - acción. INVENIO, (31-32) ,121-143.

(26) Romano, A. \& Redondo, J. (2016). Aplicación de la investigación- Acción a la planificación de la sucesión en una empresa familiar de pequeño porte. INVENIO, 19(36), 65-87.

(27) Romero, J. (2009). Dinámica interna de la empresa familiar exportadora. Revista Finanzas y Política Económica, 1(2), 53-70.

(28) Sandoval, J. \& Guerrero, D. (2010). Empresas familiares en Colombia: Hacia la construcción de un modelo de gestión comercial. Revista U.D.C.A Actualidad \& Divulgación Científica, 13(1) ,135-146. 
(29) Vélez, D., Holguín, H., De la Hoz, G., Durán, Y. \& Gutiérrez, I. (2008). Dinámica de la empresa familiar PYME: Estudio exploratorio en Colombia. Red FUNDES, Internacional.

(30) Zeron, M., Mendoza, G. \& Lara, J. (2009). Pequeñas empresas familiares y sus estrategias de negocios. Recuperado el 11 de agosto de 2018, de http://www.eumed.net/ce/2009b/fct.htm. 